\title{
Comparision of Efficacy between Thiocolchicoside and Tizanidine on Sequelae of Impacted Third Molar Removal
}

\author{
Dr. B.H Sripathi Rao ${ }^{1}$, Dr. Arvind Rao.H.T², Dr. Shruti.G.RAO ${ }^{3 *}$
}

\author{
${ }^{1}$ Professor, Head of the Department Dept. of Oral and Maxillofacial Surgery, Yenepoya Dental College, Mangalore, Karnataka India \\ ${ }^{2}$ Reader, Dept. of Oral and maxillofacial surgery, Yenepoya Dental College, Mangalore, Karnataka India \\ ${ }^{3}$ Post Graduate, Dept. of Oral and Maxillofacial surgery, Yenepoya Dental College Karnataka India
}

DOI: $10.36347 /$ sjds.2020.v07i01.005

| Received: 21.01.2020 | Accepted: 28.01.2020 | Published: 30.01.2020

*Corresponding author: Dr. Shruti. G.Rao

Abstract

Original Research Article

Surgical extraction of an impacted lower third molar involving bone removal doubtlessly causes some degree of pain, swelling, and trismus. Reducing or minimizing muscle spasm after third molar surgery would be a creditable goal, particularly if clinical healing were not compromised. Skeletal muscle relaxants are a heterogeneous group of medications used to treat two different types of underlying conditions: spasticity from upper motor neuron syndromes and muscular pain or spasms from peripheral musculoskeletal conditions including fibromyalgia, tension headaches, myofascial pain syndrome, and mechanical low back or neck pain. Thiocolchicoside and Tizanidine being centrally acting skeletal muscle relaxants are known to reduce the post-operative sequelae caused due to third molar impaction surgery. This study was conducted to compare the potent drug among the two after third molar surgery.

Keywords: Thiocolchicoside, Tizanidine, Muscle spasm, $3^{\text {rd }}$ molar surgery.

Copyright @ 2020: This is an open-access article distributed under the terms of the Creative Commons Attribution license which permits unrestricted use, distribution, and reproduction in any medium for non-commercial use (NonCommercial, or CC-BY-NC) provided the original author and source are credited.

\section{INTRODUCTION}

Third molar impaction procedure is frequently followed by complications in the mandible [1-3], including both iatrogenic (e.g., nerve injury, bone fractures, etc.) and inflammatory ones, such as dry socket, postoperative pain, delayed healing, postoperative infection, hematoma, swelling, trismus, etc[4,5]. Most of these complications are minor and the overall complication rate is generally low. Noninfection-related swelling, pain, and trismus are the three most common sequelae following removal of impacted mandibular third molars. It is important to identify the causative factors and select the suitable treatment approaches to limit clinical sequences after the removal of third molars, with the goal of reducing discomfort and increasing patient's functional capacity in the postoperative period.

Extension of the incision as well as tissue manipulation and length of the surgery could affect the entity of swelling [6] and increases the level of pain and discomfort post operatively, to the patient. These complications have been reported to have an adverse effect on the quality of life of patients undergoing third molar surgery. Trismus may result from some sort of insult to the muscles of mastication, such as opening the jaw for a period of time or having a needle pass through a muscle. Dental anaesthesia to the lower jaw often involves the needle passing into or through the medial pterygoid or the buccinator muscles. Although its harmless most of the times, but it could give rise to many constraints for the patient, including social injunctions that can cause anxiety and danger. In a few instances, lawsuits have been instigated. Therefore, it is important for clinicians to be aware of this significant condition, its primary causes, and its treatments [7].

The influence of a muscle relaxant on postoperative swelling, pain, and mouth opening limitation after third molar extraction is proved to be quite necessary. They have proved to be well suited to decrease spasticity, primarily in the treatment of muscular hypertension. The goal with these medications is a reduction of skeletal muscle spasms, relief of pain, and increased mobility of the affected muscles.

Skeletal muscle relaxants are widely used to reduce acute low back pain, various neurological conditions and also during surgical procedures and in emergency medicine to cause temporary paralysis of the muscles. Hence, both neuromuscular blockers and spasmolytics are grouped together as muscle relaxants. Several studies have been done on different muscle 
relaxants in oral surgery, post third molar impaction[8] and acute myofascial pain syndrome [9] etc. However, the results were quite satisfactory in few studies and also equally ambiguous in few others.

Thiocolchicoside and tizanidine, two centrally acting skeletal muscle relaxants are expected to reduce these post-operative complications and discomfort. The following study is based on the comparision of the efficacy of these particular skeletal muscle relaxants in the sequelae of $3^{\text {rd }}$ molar impaction.

It has been shown previously that thiocolchicoside induces muscle relaxation without any subjective or objective sedative side effect [10]. In experimental models, thiocolchicoside was also found to possess analgesic and anti-inflammatory activities, and in a pharmaco-EEG study, thiocolchicoside was also shown to be devoid of any sedation [11]. Clinical trials have demonstrated that the therapeutic efficacy of thiocolchicoside is associated with a good level of safety and sedation was rarely reported.

Previous studies in the treatment of acute low back pain, and other conditions involving painful muscle spasm have shown Tizanidine to be effective as monotherapy and in combination with non-steroidal anti-inflammatory drugs (NSAIDs)[12]. Results of the studies show that Tizanidine is effective in relieving painful muscle spasm, is safe and well tolerated. The findings confirm the significant improvement in muscle spasm and other pain parameters by using Tizanidine as a monotherapy [13-15].

Various pharmacological methods are known and being followed to reduce the pain, swelling and trismus caused due to impacted tooth removal. Skeletal muscle relaxants are one among them. Taking this into consideration, this study is planned to know and compare the efficacy of the two widely used muscle relaxants, Thiocolchicoside and Tizanidine in oral surgery, post third molar impaction surgery.

\section{MATERIALS \& METHODS}

This study entailed the use of the two different skeletal muscle relaxants for two groups of patients after the removal of mandibular impacted third molar.

\section{Source of data}

Thirty healthy adult patients between the age group of 18-30 years reported to the department of oral and Maxillofacial surgery, yenepoya dental college with impacted mandibular third molars, willing for prophylactic removal of the tooth were selected and taken up for the study.

\section{INCLUSION CRITERIA}

- Only healthy patients between $18-30$ years of age will be included in the study.

- $\quad$ Patient consenting to the study protocol.
- $\quad$ Patient having Pell \& Gregory’s position B\&C will be included in the study based on radiographic interpretation.

- Any bony impacted third molar tooth removal which lasts for more than 30minutes.

\section{EXCLUSION CRITERIA}

- Patients with systemic disease that could influence healing via metabolic bone disease, endocrinal disease would be excluded.

- Patients with any kind of past Medical or Drug histories contraindicating the use of the study medication.

- Patient with current signs and symptoms of acute infection or pain.

- Patients on use of antibiotics and or analgesics within the 48 hour period before the surgery.

- Patients who are contraindicated for radiograph.

- Pregnant and lactating mothers.

\section{METHOD AND COLLECTION OF DATA}

The patients in this study were divided into two groups Group A- Tizanidine (Sirdalud) 2mg was administered. Group B- Thiocolchicoside (Myoril) 4mg was administered.

\section{METHODOLOGY}

Written informed consent was obtained and a patient questionnaire was filled out by the investigator at least 24 hours before surgery. Demographic data such as the subject's age, gender, health history and current medications were recorded. The difficulty of the removal procedure was evaluated pre operatively according to Pedersons scale on each panoramic radiograph.

The standardized operation procedure was followed by the surgeons in the outpatient setting at the Department of Oral \& Maxillofacial Surgery under local anaesthesia (2\% lignocaine hydrochloride with adrenaline in 1:80,000 dilutions) without premedication or sedation. In our surgical procedure, hand piece used was sterilized, in a steam autoclave. Copious irrigation using cold saline or distilled water was done to prevent heating of bone and subsequent bone necrosis since; cooling the bone by way of irrigation will also reduce the postoperative pain and edema. The use of a high vacuum suction to clear the surgical site while irrigation is used cannot be over emphasized.

Buccal guttering technique was followed in our procedure. The buccal cortex contributes greatly to the strength of the mandible. Hence buccal bone removal should be kept to minimum to avoid weakening of the mandible and subsequent fracture. The bone on the buccal and the distal aspect of the impacted tooth was removed down to the level of the cervical line. Further bone removal if required was done in a manner not detrimental to the strength of mandible 
and at the same time not sacrificing the efficiency of surgery. This was achieved by drilling a deep vertical gutter alongside the buccal aspect and if required on the distal aspect of the tooth. This 'guttering method' ensured that the height of the buccal plate was maintained without weakening the mandible and at the same time adequate space was created around the tooth to permit its free movement.

Bone covering the mesial aspect of the impacted tooth was also removed by this method. Drilling in the region of the mesial surface of impacted tooth was kept to the minimum to avoid damage to the distal aspect of the adjacent second molar. While removing bone on the distolingual aspect extreme care was taken to protect the lingual nerve from bur by way of proper retraction. It is advisable not to remove any bone on the lingual aspect due to the likelihood of damage to the lingual nerve. A variety of burs were used to remove bone, but the most commonly used were the \#8 round bur and a \#703 fissure bur.

Closure was done using non resorbable 3-0 black braided silk sutures and no cold dressing in the form of ice pack were given as they are known to decrease the edema and the resultant post-operative swelling.

\section{Post-Operative Medications}

Protocol for all patients included prescription of $500 \mathrm{mg}$ of Amoxicillin every 8 hours for 5 days and $50 \mathrm{mg}$ diclofenac sodium every 8 hours for 3days. In addition to the standard medication, the Group A (15 patients) were prescribed $2 \mathrm{mg}$ tizanidine (Sirdalud) orally twice daily for 3 days and Group B (15 patients) were prescribed $4 \mathrm{mg}$ of thiocolchicoside(Myoril) orally twice daily for 3 days.

Postoperative information advised to the patients included, not to drive a vehicle or any other active physical exertions for next 3 days. Patients were recalled for follow-up on days 1, 3 and 7 after surgery.

\section{Pre-operative Parameters}

- Pain according to $10 \mathrm{~cm}$ Visual analogue scale was recorded.

- According to Amin and Laskin [29] Horizontal distance (distance between the lower attachments of the ear lobe to the corner of the mouth) and Vertical distance (Distance between the angles of the mandible to the outer canthus of the eye) were measured by means of a thread and a measuring tape.

- Maximum mouth opening (distance between the incisal edges of the upper and lower incisors) was measured.

\section{Intra Operative Parameters}

Time taken for the surgery was recorded right from the start of incision placement till the tooth was completely out of the socket.

\section{Post-Operative Parameters}

All postoperative data were recorded. At every appointment the presence of paresthesia, fatigue or infectious complications were noted, and patients were examined on the main variables of trismus, pain and the presence of swelling.

Pain intensity was assessed on a $100 \mathrm{~mm}$ visual analogue scale (VAS) by placing a mark between the end points of $0 \mathrm{~mm}$ indicating no pain and $100 \mathrm{~mm}$ indicating unbearable pain.

Extraoral swelling was assessed by measuring using a thread from tragus to the corner of the mouth (posteroanteriorly) and from lateral canthus of the eye to the lower border of mandible (superioinferiorly) on day 1, 3 and 7 posts operatively.

The maximum mouth-opening ability was assessed by measuring the maximum interincisal distance at every postoperative appointment.

On day 7 patients were asked to evaluate the effects of the medication with regard to trismus, swelling, and pain on a 5-point scale. The scale categories are: 0: poor; 1: fair; 2: good; 3: very good; and 4: excellent.

\section{RESULTS}

Following completion of the clinical study on the patients, the measurements and data taken from all the patients were tabulated for statistical studies following decoding of the drugs used. The various aspects of the studies were tabulated and compared. Following are the results and observations noted.

\section{PAIN}

On comparing the reduction in pain from $1^{\text {st }}$ post-operative day to $3^{\text {rd }}$ post-operative day, between thiocolchicoside and tizanidine, we found there was no significant difference between the two, but mean increase in pain was more in thiocolchicoside(5.533) when compared to tizanidine (5.133).

On comparing the reduction in pain from $1^{\text {st }}$ post-operative day to $3^{\text {rd }}$ post-operative day, we found that there was no significant difference between the two, but mean increase was more in thiocolchicoside(4.133) when compared to tizanidine (3.933). 
On Comparing the reduction of pain from $1^{\text {st }}$ post-operative day to $7^{\text {th }}$ POD, we found that there was no significant difference between the two, but mean increase was slightly more in thiocolchicoside (2.866) when compared to tizanidine (2.733)

\section{SWELLING}

On comparing the percentage of swelling from pre-operative day to $1^{\text {st }}$ post-operative day between thiocolchicoside and tizanidine, we found that the swelling was more in tizanidine(127.46) when compared to thiocolchicoside (122.13) in horizontal reading. In vertical reading also, swelling was more in tizanidine(126.06) when compared to thiocolchicoside (121.06)

On comparing the percentage of swelling from $1^{\text {st }}$ post-operative day to $3^{\text {rd }}$ post-operative day of thiocolchicoside and tizanidine, we found that the swelling was more in tizanidine (125.33) when compared to thiocolchicoside (120.4) in horizontal reading. In vertical reading also, swelling was more in tizanidine (124.2) than thiocolchicoside (118.66).

From $3^{\text {rd }}$ post-operative day to $7^{\text {th }}$ postoperative day of thiocolchicoside and tizanidine, there was no significant difference between the two drugs in reduction of the swelling. However, swelling was more in tizanidine(122.86) when compared to thiocolchicoside (116.86) in horizontal reading. In vertical reading also, swelling was more in tizanidine (122.13) than thiocolchicoside (116).

\section{TRISMUS}

On comparing the mean value of the mouth opening in patients from pre-operative day to $1^{\text {st }}$ postoperative day, we found that there was no significant difference between the two, but the mean was more for tizanidine(32) when compared to thiocolchicoside (29.33).

On comparing the mean value from $1^{\text {st }}$ postoperative day to $3^{\text {rd }}$ post-operative day, we found that there was no significant difference between the two, but the mean was more for tizanidine(38.33) when compared to thiocolchicoside (34.53).

On comparing the mean value of the mouth opening in patients from $3^{\text {rd }}$ post-operative day to $7^{\text {th }}$ post-operative day, we found that there was no significant difference between the two , but the mean was more for tizanidine(42.8) than thiocolchicoside (38.33).

\section{DISCUSSION}

Innumerable patients undergo surgical procedures in the department of oral and maxillofacial surgery throughout the world each year. Impaction of the mandibular third molar is a common condition giving rise to symptoms which necessitate its removal.
Literature shows that the most common complications after surgical removal of mandibular third molars are pain, trismus and swelling $[16,17]$.

The factors contributing to the post-operative morbidity are many, but the most important one is the trauma from bone cutting as the procedure involve significant bone cutting, which was carried out by rotary cutting instruments (like surgical bur). The major step in removal of an impacted $3^{\text {rd }}$ molar is to remove the bone around the impacted tooth. The amount of bone removal varies with the depth of impaction. As stated above in the inclusion criteria, our study included teeth with pell \& Gregory position B \& C, and also any bony impacted tooth which lasts for more than 30 minutes. The bone removal around the impacted tooth can be accomplished either by the use of a bur, or a chisel and mallet or a combination of the two methods. Whatever may the method used (which may be of individual preference), the aim is to remove sufficient amount of bone to free the tooth from obstruction and to provide a point of application for the elevator.

Electric drill (micromotor and handpiece) is considered the instrument of choice in the present day impaction surgery. It has the advantage of rapid bone cutting with minimal discomfort to the patient. "Guttering method was used in our study which is a technique in which a deep vertical gutter using a bur is made alongside the buccal aspect and if required on the distal aspect of the tooth too. Thoma[18] and Archer[19] using buccal bone cutting, mentioned that swelling was a known complication of third molar surgery. The presence of swelling or infection causes spasm of muscle leading to trismus. Bleeding can be attributed to two factors primarily due to the dislodgment of clot and secondary due to infection [1820].

Traditionally post-operative sequelae are treated by health professionals, using various drugs like analgesics and anti-inflammatory drugs. Pharmacological strategies for minimizing the clinical manifestations of surgical trauma are often directed towards blocking the formation or inhibiting the effects of the biochemical mediators of acute inflammation.

Pain, being subjective criteria is difficult to assess. It is difficult to measure pain, partly because it is usually accompanied by another sensation and partly because the reaction component affects the judgement of pain regardless of the intensity of the stimulus. Pain after minor oral surgical procedure, is expected after local anaesthetic action has weaned off, and previous trials conducted by Linden Seymour et al. [21] have concluded that maximum intensity of pain occurs in the first 12 hours. The post-operative pain experience in patients depends on a variety of factors and it will increase between the post-operative days 1 and 3 after 
which symptoms subside gradually within one week [22].

Linden et al. [21] described that the visual analogue scale was more sensitive and a better measure of the magnitude of pain. Investigations using visual analogue scale showed that patients preferred a horizontal scale as opposed to vertical scale. In our study we used a verbal analogue scale to measure the intensity of pain at various stages in the post-operative period. The recording of pain was very simple; however accuracy of intensity of pain given by each patient might slightly differ depending upon the pain threshold of each individual. Hence there can be a marginal difference in the prediction of intensity of pain.

After analysing the pain intensity in both the groups of patients receiving thiocolchicoside as well as tizanidine, no significant difference was noted between both the drugs in reducing the intensity of pain. However a marginal percentage difference was noted where patients receiving tizanidine had a slight edge over the other group of patients receiving thiocolchicoside. However, this difference was not very significant. Pain was high on First post-operative day and gradually decreased from post-operative day 3 to post-operative day 7 in both the groups.

Post-operative swelling is due to the combined action of the inflammatory mediators which induce vasodilation and increased vascular permeability. Swelling is may be because electric driven instruments generates a certain amount of heat and onwards transmission force enough to drive the bone particles deeper to bony canaliculi [23]. Another reason for swelling is, may be because of the inability to achieve complete sterilization of bur and hand piece assembly, which lead to cross infection and brushing of surrounding tissues [20]. It is likely that the postoperative swelling contributes considerably to the pain because of increased tension in the tissues.

An absolute determination of an increase in volume of the maxillofacial area both intraorally and extraorally in a large group of patients cannot be achieved with simple mean. In some investigations, the swelling of the cheek is measured directly by means of a standard plastic tape measure or by callipers. The accuracy is rather variable. Using ultrasound, however, it is possible to assess directly the increase in the distance between the mucosa and the skin surface produced by the edema. Besides ultrasonography, alternative methods used to document soft tissue swellings are visual analog scales, photographs and facebows[24-27]. The disadvantage is that these methods offer merely an indirect assessment of swelling on the skin surface. Some investigators also regard computed tomographic documentation as an objective method of determining post-operative soft tissue swelling. However, this method is too complicated and expensive for a routine post-operative use to check the progress of swelling.

According to Oliver Ristow et al. [27], Swelling was assessed with a five-line measurement using a standard plastic tape measure (accuracy 0.5 $\mathrm{mm}$ ) placed in contact with the skin:

(LINE A) - Most posterior point tragus to most lateral point lip commissure

(LINE B) - Most posterior point tragus to pogonium

(LINE C) - Most posterior point tragus to lateral canthus of the eye

(LINE D) - Lateral canthus of the eye to most inferior point angle of the mandible

(LINE E) - Most inferior point angle of the mandible to middle of the nasal bone

The mean sum of the five measurements was taken as the final assessment of swelling [28]. In our study, we have used the technique advocated by Amin and Laskin[29] for measuring the extra oral swelling in horizontal and vertical directions. It is a very simple method to measure the extra oral swelling between the fixed points which are, horizontally from tragus of the ear to the corner of the lip and in vertical direction from lateral canthus of the eye to the angle of the mandible, but this technique fails to give any indication regarding mediolateral dimension of intraoral swellings that occurs following $3^{\text {rd }}$ molar surgeries.

Both the horizontal and vertical dimensions of the swelling increased in patients who were administered tizanidine when compared to the group which were given thiocolchicoside. Swelling in the horizontal dimension increased slightly from postoperative day 1 to post-operative day 3 with respect to the group which was administered thiocolchicoside and then gradually decreased from post-operative day 3 to post-operative day 7 . Whereas, vertical readings of the swelling decreased gradually from post-operative day 1 to 7 in both the groups.

A prophylactic course of antibiotics was given to all the patients to avoid any possibility of infection which otherwise might contribute in exaggerating the post-operative sequelae.

Trismus limits the mouth opening in an attempt to prevent additional trauma or pain. In addition to this, pain, inflammatory edema and trauma to muscle also contribute to the severity of trismus. If we are able to control the above factors by pharmacological means, trismus will improve with the reduction of pain and inflammatory edema. However, in some cases it might persist for a longer period which could be due to direct trauma to the muscle fibres or due to secondary infection. 
Most clinicians endorse the idea that muscle pain induces some degree of muscle hyperactivity, which can in turn cause more pain. Although pain in a local area does mandatorily induce tightening of the surrounding muscles, sometimes the opposite is true. Pain may inhibit rather than facilitate reflex contractile activity, so the decision to treat a patient with a muscle relaxant should not be based solely on pain but also on physical signs that include muscle tightness and/or taut bands. Unfortunately, there is very little evidence-based medicine available to guide the choice of a medication for an acute, uncomplicated musculoskeletal disorder since only a limited number of high qualities, randomized controlled trials, provide evidence of the effectiveness of muscle relaxants. As mentioned previously, the distinction between antispasticity drugs and muscle relaxants is based not on their site of action (e.g., central vs. peripheral) since most of these drugs act centrally, but on their relative ability to suppress upper and lower motor neuron activity., muscle relaxants are typically used in conditions with minor skeletal muscle spasms such as a tight, sore, and stiff muscle since they are better tolerated, with relatively fewer adverse events [30].

In our study, we have used two centrally acting skeletal muscle relaxants for their effects on decreasing muscle spasm and trismus which causes reduced mouth opening. Trismus is basically measured by noting the maximum inter incisal distance. This can be measured either by a scale or a vernier calliper.

The Group which was administered thiocolchicoside exhibited less mouth opening when compared to the group with tizanidine. The difference gives us a clear indication that tizanidine decreases the trismus significantly. Mouth opening improved effectively over $3^{\text {rd }}$ and $7^{\text {th }}$ post-operative day in all the patients irrespective of the drug administered.

\section{CONCLUSION}

In our study we have compared the efficacy between two skeletal muscle relaxants, Thiocolchicoside and Tizanidine in reducing the postoperative sequelae such as pain, swelling and trismus. We have arrived to a conclusion that there is no significant difference between the two drugs in reducing the pain, swelling and trismus. Both Tizanidine and Thiocolchicoside are proved to be helpful in reducing the Post-operative discomfort levels in the patients after third molar impaction surgery. For a conclusive report, a further study with a large number of clinical cases with different clinical situations should be used to authenticate these values.

\section{REFERENCES}

1. Nordenram A. Postoperative complications in oral surgery. A study of cases treated during 1980. Swedish dental journal. 1983;7(3):109-14.
2. Heng CK, Badner VM, Clemens DL, Mercer LT, Mercer DW. The relationship of cigarette smoking to postoperative complications from dental extractions among female inmates. Oral Surg Oral Med Oral Pathol Oral Radiol Endod. 2007; 104:757-762.

3. El-Soud NA, El Shenawy H. A randomized double blind clinical study on the efficacy of low level laser therapy in reducing pain after simple third molar extraction. Maced J Med Sci. 2010;3:303306

4. Bui CH, Seldin EB, Dodson TB. Types, frequencies, and risk factors for complications after third molar extraction. J Oral Maxillofac Surg. 2003; 61:1379 1389 .

5. Peñarrocha M, Sánchis JM, Saez U, Gay C, Bagán JV. Oral hygiene and postoperative pain after mandibular third molar surgery. Oral Surg Oral Med Oral Pathol Oral Radiol Endod. 2001; 92:260-264.

6. Strategies used to inhibit postoperative swelling following removal of impacted lower third molar Francesco Sortino1 and Marco Cicciù2 Dent Res J (Isfahan). 2011 Oct Dec; 8(4): 162-171

7. Dhanrajani PJ and Jonaidel O. Trismus: Aetiology, Differential Diagnosis and Treatment; Dent Update. 2002; 29: 88-94

8. Prediction of postoperative facial swelling, pain and trismus following third molar surgery based on preoperative variables Thiago de Santana-Santos, Jadson A. de Souza-Santos, Paulo R. MartinsFilho, Luiz C. da Silva, Emanuel D. de Oliveira e Silva, and Ana C. Gomes Med Oral Patol Oral Cir Bucal. 2013 Jan; 18(1): e65- e70. Published online 2012 Dec 10.

9. Neupert III EA, Lee JW, Philput CB, Gordon JR. Evaluation of dexamethasone for reduction of postsurgical sequelae of third molar removal. Journal of oral and maxillofacial surgery. 1992 Nov 1;50(11):1177-82.

10. Patat A, Klein MJ. Effects of acute and repeated doses of two muscle relaxants chlormezanone and thiocolchicoside, on vigilance and psychomotor performance of healthy volunteers.Hum Psychopharmacol. 1991; 6:285-92.

11. Janbroers JM. Review of the toxicology, pharmacodynamics and pharmacokinetics of thiocolchicoside, a GABA-agonist muscle-relaxant with anti-inflammatory and analgesic actions. Acta Ther. 1987; 13:221-50.

12. Role of muscle relaxant ( tizanidine) in painful muscle spasm" by Riaz ahmed sheikh, Khurrum siddique, Shirin mirza,Syeda fizza tauqir. Ann. Pak. Inst. Med.sci.2010;6(1):20-23.

13. Berry H, Hutchinson Dr: A Multicentre PlaceboControlled Study in Practice to Evaluate Efficacy and Safety of Tizanidine in acute low back pain. J Int Mad Res.16:75, 1988.

14. Hutchinson DR, Daniels F, Jaga P: A Multinational Study to Evaluate the Effectiveness and 
Tolerability of Tizanidine in the Treatment of Acute Painful Muscle Spasms. Br J Clin Res. 1:33, 990.

15. Garcia Rosas R, Escobedo Brito V: National Multicentre Study for the Evaluation of Efficacy and Tolerability of Tizanidine in the Management of Painful Muscle Spasms. J Int Med Res. 19:166, 1993.

16. Edward A, Jesse W.L, Christine B.P and Gordon J.R. Evaluation of dexamethasone for reduction of post-surgical sequelae of third molar removal. J oral maxillofacial Surg. 1992; 50:1177-1182.

17. Forsgren hanz, Heimdahl A, Johansson $\mathrm{b}$ and Krekmanov L, Effect of application of cold dressings on the post-operative course in oral surgery. Int J Oral Maxillofac surg. 1985; 14:223228.

18. Thoma KH. Oral Surg. In: Bioanatomy. 4 th ed, Vol. 1. St Louis: C. V. Mosby Co. 1963: 29.

19. Archer WH. Oral and Maxillofacial Surgery. 5 th ed, Vol. 1. USA: W.B. Saunders Company; 1976.

20. Szmyd L, Hester WR. Crevicular depth of the second molar in impacted third molar surgery. J Oral Surg. 1963;21:185-9.

21. Linden, Abraham, Matheny, Kaplan: pain operative pain and local anaesthesia.J. of periodontology 1986;57(1C): 637-641

22. Raisian S, Fallahi H,badakhshan 1 and zandian D. A randomised double blind controlled trial comparing ibuprofen versus ibuprofen plus Acteaminophen plus caffeine for pain control after impacted third molar surgery. Open Journal of stomatology. 2012;2(2): 110-115.
23. Vibha Singh, Khonsao Alex, R. Pradhan, Shadab Mohammad, Nimisha Singh | European Journal of General Dentistry. 2(1): (2013).

24. Cooper SA,Needle S.E, Kruger GO, comparative Analgesic potency of aspirin and ibuprofen. J.Oral Surg. 1977;35:889-903

25. Forbes AJ,bowser M.W, calderazzo J.P,Foor V.M. An evaluation of the analgesic efficacy of 3 opiod analgesic combinations in post-operative oral surgery pain. J of oral surgery. 1981;39:108-112

26. Forbes J.A, butterworth GA, Kehm CJ, Grodin CD, Yee JP, Beaver Wt. Two clinical evaluation of ketorolac tromethamine in post-operative oral surgery pain. Clin.pharmac and therap. 1987;41:162

27. Frame J.A, Evans C.R.H, Flaum G.R, Langford R, Rout D.G.J. A comparision of ibuprofen and dihydrocodeine in relieving pain following wisom teeth removal. British dental journal. 1989; 166:121-124.

28. Ristow O, Pautke C, Kehl V, Koerdt S, Schwärzler K, Hahnefeld L, Hohlweg-Majert B. Influence of kinesiologic tape on postoperative swelling, pain and trismus after zygomatico-orbital fractures. Journal of Cranio-Maxillofacial Surgery. 2014 Jul 1;42(5):469-76.

29. Amin M.M,Laskin M. Prophylactic use of Indomethacin for prevention of post-surgical complications after removal pf impacted third molars. OS: OM:OP. 1983;55(5):448-451.

30. Clark GT, Sahai-Srivastava S. Skeletal muscle relaxants and antispasticity drugs for orofacial pain disorders. Orofacial Pain. 2012 Feb 17:115. 